\title{
Sextant
}

Revue de recherche interdisciplinaire sur le genre et la sexualité

$37 \mid 2020$

Le rideau déchiré

\section{La guerre de mouvement des catholiques}

Les Colloques internationaux de sexologie de Louvain (1959-1974)

Cécile Vanderpelen-Diagre

\section{(2) OpenEdition}

12 Journals

Édition électronique

URL : https://journals.openedition.org/sextant/333

DOI : $10.4000 /$ sextant.333

ISSN : 2795-8736

Éditeur

Éditions de l'Université de Bruxelles

\section{Édition imprimée}

Date de publication : 1 décembre 2020

Pagination : 99-114

ISBN : 978-2-8004-1744-8

ISSN : $1370-267 X$

\section{Référence électronique}

Cécile Vanderpelen-Diagre, «La guerre de mouvement des catholiques », Sextant [En ligne], 37 | 2020, mis en ligne le 01 novembre 2021, consulté le 24 janvier 2022. URL : http://journals.openedition.org/ sextant/333; DOI : https://doi.org/10.4000/sextant.333

\section{(c) (i) (8)}

La revue Sextant est mise à disposition selon les termes de la Licence Creative Commons Attribution Pas d'Utilisation Commerciale - Partage dans les Mêmes Conditions 4.0 International. 


\title{
La guerre de mouvement des catholiques Les Colloques internationaux de sexologie de Louvain (1959-1974)
}

\author{
Cécile VANDERPELEN-Diagre
}

Entre 1959 et 1978, des experts en sexualité venus du monde entier se rassemblent aux Colloques internationaux de Louvain. Initiative du cardinal Suenens, évêque auxiliaire de Malines nommé archevêque en 1962, ces rencontres sont une tentative de fonder une institution capable de répondre aux besoins de l'Église et du monde académique catholique sur les questions de sexualité. Au départ satellite de l'Université catholique de Louvain, les Colloques s'y intègrent peu à peu. Durant les quinze années de leur existence, ils rassemblent essentiellement des médecins, des psychologues et des théologiens venus principalement de France et de Belgique, mais également, pour un nombre appréciable, par ordre décroissant, de GrandeBretagne, des États-Unis, d'Italie, des Pays-Bas, du Canada et de Suisse ${ }^{1}$. Ils sont un lieu d'échanges entre experts qui cherchent à trouver une sorte de lingua franca sur les questions de sexualité dans le but d'énoncer un message univoque aux fidèles qu'il s'agit d'éduquer. Ces rencontres marquent aussi une étape importante dans l'histoire de la gestion par l'Église de la sexualité. Alors que naguère, elle envisageait ce domaine comme ressortissant principalement à la théologie et à la pastorale, elle puise désormais dans les sciences médicales, biologiques, neurologiques et psychologiques des outils pour comparaître dotée d'une expertise élargie.

L'histoire de ces Colloques est intéressante pour l'étude de la sexologie catholique dans une période charnière de l'histoire de la sécularisation des sciences « catholiques », ou plus exactement de l'éloignement de la science catholique de l'autorité cléricale. L'historiographie sur l'évolution des politiques de l'Église en matière de sexualité (pastorale à destination des couples, aide au mariage, action

${ }^{1}$ L. Boute, « Les Colloques de Louvain de Mgr Suenens (1959-1974) : contributions aux débats romains sur la morale conjugale ? », [2016], article inédit. 
catholique) est bien fournie. Pour ne parler que de la France, grâce aux travaux de Martine Sevegrand, Anne-Marie Sohn ${ }^{2}$ et Agnès Desmazières ${ }^{3}$, l'histoire des médecins, psychiatres et psychanalystes catholiques est connue, tant pour ce qui est de leur engagement que de leurs pratiques. Enfin, la place et l'influence des catholiques dans l'éducation sexuelle ont été très bien étudiées par Alain Giami ${ }^{4}$. Ces travaux ont montré, notamment, les tensions entre morale, ecclésiologie, théologie et pastorale. Les Colloques internationaux éclairent une autre dimension : l'articulation constamment renégociée entre les contraintes religieuses, scientifiques et idéologiques. Se voulant un laboratoire international, ils sont enfin une caisse de résonnance d'enjeux plus larges qui concernent la régulation des naissances et les normes en matière de sexualité et de genre qui deviennent à cette époque des questions débattues à une échelle globale. Comme cet article vise à le montrer, l'Église se veut en effet une actrice incontournable dans la circulation internationale des savoirs scientifiques, des financements de la recherche, des pratiques et des normes reproductives et sexuelles. Sur ces questions comme sur d'autres, durant la guerre froide, elle épouse volontiers le discours occidental libéral de la « liberté » qu'elle adapte toutefois à ses propres besoins. L'analyse fine des actes de ces Colloques sur laquelle se base cet article vise à montrer qu'ils sont des laboratoires où se discute l'amplitude de l' « adaptation ».

\section{Un conseil d'experts en sexualité autour du Saint-Siège}

Selon l'historien Léo Declerck, Léon-Joseph Louis Suenens, alors évêque auxiliaire du cardinal Joseph-Ernest Van Roey, découvre l'importance de la planification des naissances en 1958 grâce à son amie l'Irlandaise Veronica O'Brien, avec laquelle il est engagé dans la fondation et la promotion de la Légion de Marie ${ }^{5}$. La même année, la résolution de la guerre scolaire qui secouait la Belgique depuis des décennies et dans laquelle Suenens s'était fort investi lui laisse le champ libre pour s'engager dans un combat plus porteur au niveau international, projet fondamental s'il veut monter les échelons de la hiérarchie. Lors de la Conférence mondiale des médecins catholiques à Bruxelles le 28 juillet 1958, il lance un appel aux médecins et chercheurs qu'il invite à étudier les pratiques de régulation des naissances afin d'améliorer la situation des

${ }^{2}$ A.-M. SoHN, « Les catholiques français entre abstinence et "apaisement de la concupiscence" ", Sexual Cultures in Europes. Themes in sexuality, edited by Franz Eder, Leslie Hall, Gert Hekma, 1996, p. 233-254 et M. SEvegrand, Les Enfants du bon Dieu. Les catholiques français et la procréation au XX siècle, Paris, Albin Michel, coll. « Histoire », 1995.

${ }^{3}$ A. Desmazières, « La psychanalyse entre médiatisation et censure. La morale sexuelle de Marc Oraison en procès (1955-1966) », Archives de sciences sociales des religions, 163/3, 5 décembre 2013, p. 123-142.

${ }^{4}$ A. Giami, "Une histoire de l'éducation sexuelle en France : une médicalisation progressive de la sexualité (1945-1980) », Sexologies, 16/3, $1^{\text {er }}$ juillet 2007, p. 219-229, coll. « Une histoire de la sexologie française / A history of French sexology ».

${ }^{5}$ L. DeCLERCK, « Le cardinal Suenens et la question du contrôle des naissances au Concile Vatican II », Revue théologique de Louvain, 41, 2010, p. 499-518. 
croyants. Il en appelle aussi à la création de centres d'études sur la sexologie dans les universités catholiques ${ }^{6}$ :

Il est des problèmes cruciaux - je songe tout particulièrement aux problèmes de morale conjugale - que le prêtre ne peut résoudre sans vous, sans l'aide de vos recherches et de votre appui médical et moral. [...] Nous n'avons pas le droit de demander aux hommes d'observer la loi sans faire en même temps tout ce qui est en notre pouvoir pour rendre l'obéissance possible [...]. Il est des péchés d'inertie et de paresse intellectuelle qui pèseront bien plus lourd au jour du jugement dernier que les péchés de faiblesse ${ }^{7}$.

C'est animé par cet objectif que Suenens réunit en 1959 le premier Colloque sur le thème " Maîtrise sexuelle et maîtrise de la nature », lequel a pour but d'

étudier les aspects médicaux de la fertilité en vue de rendre possible l'intimité conjugale lorsqu'un espacement des naissances s'impose pour des motifs médicaux, psychologiques ou économiques, et étudier les aspects scientifiques de la maîtrise sexuelle. Son Excellence demande aux participants de se limiter autant que possible aux aspects scientifiques de ces questions, les aspects moraux ou pédagogiques qu'elles pourraient soulever devant être éventuellement étudiés par ailleurs ${ }^{8}$.

En dépit de ces prétentions scientifiques, les Colloques ont lieu dans un cadre privé, à huis clos, dans la maison du gynécologue-obstétricien Joseph Schokaert, fils de Ruffin Schokaert, également gynécologue-obstétricien très actif, pendant l'entredeux-guerres, avec le médecin bruxellois Raoul De Guchteneere, dans le combat contre le néomalthusianisme ${ }^{9}$. Lors des Colloques, une messe est célébrée chaque jour et les médecins ne semblent pas particulièrement attachés à l'idée de ne poursuivre qu'un but purement scientifique. Pour Guchteneere, l'essentiel doit rester de propager la méthode Ogino-Knauss. Quant à la pilule, alors appelée sous le nom générique d'« inhibiteur d'ovulation », il la déclare illicite ${ }^{10}$.

Pour Suenens, ces Colloques servent d'une part à récolter des données scientifiques et médicales afin d'enrichir et légitimer sa propre expertise, d'autre part à créer un réseau international d'experts catholiques sur les questions de sexualité. Outre de nombreux Français, des Italiens et des Anglais sont invités et se montrent très réactifs lors des débats.

${ }^{6}$ L'Institut des sciences familiales et sexologiques (ISFS) est créé à l'Université catholique de Louvain (UCL) en 1961. Il aura des connexions privilégiées avec le Centre international Cardinal Suenens (CICS) créé deux ans plus tard et l'aidera pour l'organisation des Colloques ; il en deviendra l'organisateur après la suppression du Centre en 1978. Fonds de l'ISFS, boîte 13, Archives de l'UCL.

7 L.-J. Suenens, "Christianisme et santé », extrait des Collectanea Mechliniensia, novembre 1958, p. 10.

${ }^{8}$ Colloque de Louvain, 30 et 31 mai 1959, p. 1. Les Colloques n'ont pas été édités, mais ils étaient retranscrits. Une collection complète des procès-verbaux tapuscrits se trouve aux Archives de l'Université catholique de Louvain (cote FI 69).

9 R. Christens, « De orthodoxie van het zaad», in K. Wils (dir.), Het lichaam, Louvain, Universitaire Pers Leuven, 2001, p. 241-245.

${ }^{10}$ Colloque de Louvain, 30 et 31 mai 1959, p. 12. 
En 1960, Suenens publie un ouvrage portant le titre du sujet abordé les trois premières années des Colloques, Amour et maîtrise de soi. La thèse qu'il y développe consiste à prouver que l'homme, contrairement aux animaux, est capable de contrôler sa sexualité, soit par la continence, soit par l'étreinte réservée. Cette dernière pratique, aussi appelée coitus reservatus, consiste, lors de relations sexuelles, à une pénétration qui n'est pas suivie d'éjaculation ${ }^{11}$. La charge de la preuve provient des déclarations fournies par la psychanalyse, la biologie et la psychophysiologie.

Le sexe de l'homme se situe dans le cerveau plus encore que dans les hormones. Le cerveau peut orienter et contrôler bien plus qu'on ne le pense couramment. L'homme n'a pas d'instincts au sens animal, mais des réflexes physiologiques pénétrés d'humanité. La sexualité humaine est cérébralisée, et dès lors dépendante du centre de commandement des réflexes. Le comportement sexuel de l'homme dépend, pour une très large part, de réactions non innées, mais acquises, de réflexes conditionnés, d'habitudes reçues du milieu et transmises. La réflexologie est venue montrer, par l'exemple de l'accouchement sans douleur, que la maîtrise sur les réflexes va plus loin qu'on ne l'imaginait communément. Si la physiologie du plaisir n'est pas la même que celle de la douleur, l'analogie mérite pourtant d'être relevée, et incite à poursuivre les recherches dans une direction sinon identique du moins parallèle ${ }^{12}$.

L'année même de sa parution en français, l'ouvrage, nanti d'une préface du cardinal Tardini, est traduit en allemand. Les années suivantes, il paraît en anglais, espagnol et italien. C'est que le livre répond à un vrai besoin. Lors du Colloque de Louvain de 1960, un médecin belge qui parle au nom de ses confrères explique qu'il n'y a de leur part « ni indifférence vis-à-vis de ces problèmes ni ignorance scientifique, mais un profond désarroi quant aux fondements mêmes de la loi morale, désarroi aggravé par les interprétations souvent divergentes des moralistes $\gg{ }^{13}$.

$\mathrm{Au}$ centre de l'ouvrage de celui qui devient en 1961 archevêque de MalinesBruxelles se trouve l'idée que tout le travail de l'Église et des médecins doit reposer sur l'éducation à la sexualité. Aux médecins, il revient de donner les moyens de rendre plus facile aux chrétiens l'observance de la loi divine. Il leur revient aussi de participer à la grande entreprise sociale qui consiste à répandre le discours sur les bonnes pratiques à utiliser par les croyants ${ }^{14}$. Dans un premier temps, les Colloques servent à récolter des informations sur la manière dont chaque pays s'y prend dans cette tâche. Dans la plupart des pays représentés (France, Italie, Belgique, Angleterre), ce sont les centres pastoraux de préparation au mariage et d'accompagnement à la vie conjugale et familiale qui diffusent la propagande. L'Italie semble plus avancée puisqu'elle dispose depuis 1959 d'un Centro italiano di Sessuologia étudiant les problèmes médico-moraux en matière sexuelle, soit par questionnaire, soit par contact direct avec les patients (avec établissement systématique de fiches médicales). Son directeur, le docteur Giacomo Santori, et Olindo La Pietra, le secrétaire national des

${ }^{11}$ M. SEVEGRAND, op. cit., p. 114 et s.

12 L.-J. Suenens, Amour et maîtrise de soi. Un problème crucial, [Paris-Bruges], Desclée de Brouwer, 1960, p. 11.

${ }^{13} \mathrm{II}^{\mathrm{e}}$ Colloque de Louvain, 28 et 29 mai 1960, Maîtrise de soi et Maîtrise de la nature, p. 5.

${ }^{14}$ Ibid., p. 7. 
médecins catholiques, témoignent de l'aide et du soutien qu'ils ont reçu de la part de prêtres et de religieux, mais également de profondes réticences. L'étiquette de « sexologie » déplaisant à certains membres du clergé, le centre a renoncé à l'adjectif « catholique ${ }^{15}$.

L'adjectif n'est pas repris non plus dans la dénomination de l'Institut des sciences familiales et sexologiques créé sous les auspices de Suenens en 1961. L'objectif de l'Institut, satellite de l'Université catholique de Louvain, est d'améliorer la coopération entre le monde médical et le clergé. Ses membres étudient les questions sexuelles dans leurs dimensions médicales, psychologiques, biologiques et théologiques. Quelques années plus tard, le cardinal se souviendra :

Lorsque nous avons commencé, il y a 11 ans, il était presque inconcevable d'aborder un tel sujet; c'est pourquoi, procédant à un léger camouflage, nous avons appelé notre institut « Institut des sciences familiales et sexologiques », le dernier mot sonnant mal, il fallait un petit drapeau d'avant-garde, une petite bannière pour que la procession puisse suivre ${ }^{16}$.

Notons qu'il faudra attendre 1966, soit sa huitième édition, pour que le Colloque s'intitule « international de sexologie ». Pourtant, dès 1961, plusieurs participants ont insisté sur la dénomination du Centre ou de l'école à créer :

[I]1 ne faut pas avoir peur du mot Sexologie. Ainsi les étudiants et les malades sauront où s'adresser. Pour les universités catholiques étrangères, ce sera un exemple ; enfin un tel institut catholique pourra s'opposer aux centres qui à l'étranger limitent la sexologie à la [d]iffusion des méthodes anticonceptionnelles ${ }^{17}$.

C'est que, comme le dit le docteur La Pietra,

la sexologie démarre en tant que science, mais dès à présent constitue un apostolat. Très peu de médecins y sont préparés en Italie. Y parler d'autre part de sexologie provoque des réflexes conditionnés négatifs ${ }^{18}$.

À la création de cet institut s'arriment la question de la place de l'éducation sexuelle et de la sexologie à l'université et par ricochet celle de la formation d'un personnel compétent. À l'Université catholique de Louvain, dans un premier temps, la morale sexuelle est intégrée dans le cours de "Question de sciences religieuses » obligatoire dans toutes les facultés. Les membres des Colloques de Louvain souhaitent toutefois qu'un cours spécifique soit créé, voire une chaire. S'ensuivent de longs débats pour définir la discipline de la sexologie. Tous s'accordent pour établir qu'elle est interdisciplinaire et qu'elle doit avoir une assise académique autonome par rapport aux facultés existantes. Une autre question importante est son rayonnement international.

${ }^{15}$ Ibid., p. 11.

${ }^{16}$ Actes $d u X I^{\mathrm{e}}$ Colloque international de sexologie, Louvain, 30-31 mai et $1^{\mathrm{er}}$ juin 1969, Orientations anciennes et récentes concernant les structures du comportement sexuel $d u$ couple, Centre international Cardinal Suenens, Centre international de documentation et de recherches familiales et sexologiques, Louvain, 1969, p. 217.

${ }^{17}$ III $^{\mathrm{e}}$ Colloque de Louvain, 27 et 28 mai 1961, p. 22.

${ }^{18}$ II $^{\mathrm{e}}$ Colloque de Louvain, 28 et 29 mai 1960, p. 11. 


\section{Les enjeux internationaux d'une sexologie catholique}

Il y a urgence pour les participants du Colloque de créer un secrétariat pour un organisme international de sexologie. L'influence de l'International Parenthood Federation (créée en 1952) sur l'Unesco et sur les gouvernements inquiète au plus haut point. La limitation des naissances a quitté le terrain de la pratique intime ou des politiques nationales natalistes et devient à l'époque une question internationale au sujet de laquelle le Vatican cherche à se positionner ${ }^{19}$. Certains participants des Colloques de Louvain estiment que le futur secrétariat devrait même se situer auprès du Vatican, puisqu'il s'agit de problèmes à échelle mondiale ${ }^{20}$. On touche ici à un enjeu majeur pour le Saint-Siège de l'après-guerre qui est d'asseoir un pouvoir d'influence dans « l'ordre mondial ». Il faut pour cela qu'il ait la capacité d'intervenir réellement dans le jeu de mise en place de la globalisation ${ }^{21}$.

Mais agir au niveau international suppose d'avoir une ligne doctrinale claire. C'est pour la définir que Jean XXIII rassemble en mars 1963 la Commission pontificale pour l'étude de la population, de la famille et des naissances. Suenens en est l'une des chevilles ouvrières, si pas l'un des initiateurs, et il cherche à y faire entendre son plaidoyer pour un adoucissement du dogme en matière de morale conjugale, aspiration de très nombreux catholiques ${ }^{22}$. L'histoire des tensions qui ont fracturé cette commission, opposant les partisans d'une révision de l'encyclique Casti Connubii dans le sens d'une ouverture et les opposants à tout changement, est bien connue et nous n'y reviendrons pas $\mathrm{ici}^{23}$. On retiendra que, vraisemblablement sous l'influence de Suenens, de nombreux membres de cette Commission sont également des participants très réguliers des Colloques de Louvain : l'obstétricien et endocrinologue belge Jacques Ferin, le théologien et prêtre belge Pierre de Locht, le prélat américain John Aloïs Marshal, le dominicain suisse Henri de Riedmatten et les médecins français Marcel Gauderoy et Charles Rendu, pour ne citer que les plus assidus.

Deux ans plus tôt (1965-1966), l'archevêque s'était doté d'une institution faisant directement du lobbying auprès des instances internationales : le Centre Cardinal Suenens. À sa tête, il plaçait Robert Volcher, criminologue de formation, qui dirigeait déjà l'Institut et qui devient la cheville ouvrière des Colloques

${ }^{19}$ M. Connelly, Fatal Misconception. The Struggle to Control World Population, Cambridge, Londres, The Belknap Press of Harvard University Press, 2008.

${ }^{20}$ IV $^{\mathrm{e}}$ Colloque de Louvain, 26 et 27 mai 1962, Organisation des bureaux de consultation matrimoniale, p. 23.

${ }^{21}$ A. D. Hertzke, "The Catholic Church and Catholicism in Global politics », in J. HAYNes (dir.), Routledge Handbook of religion and politics, Londres/New York, Routledge, 2009, p. 48-63 ; G. SHANI, "Transnational religious actors and international relations », in J. HAYNeS (dir.), Routledge Handbook of religion and politics, Londres/New York, Routledge, 2009, p. 308-322 et J. CASANOva, " Globalizing Catholicism and the return to a "universal church" ", in S. H. Rudolph et J. Piscatori (dir.), Transitional Religion and Fading States, Boulder, Westviewpress, 1997, p. 121-143.

22 L. DeCleRCK, op. cit.

${ }^{23}$ Voir notamment : R. MCCloRY, Rome et la contraception : Histoire secrète de l'encyclique Humanae Vitae, Paris, Éditions de l'atelier, Éditions ouvrières, 1998. 
internationaux de sexologie ${ }^{24}$. Le Centre est divisé en trois départements dévolus : 1. à la morale ; 2. à la biologie, à la neurologie, à la neurophysiologie et aux aspects " pathologiques et normaux »; 3. à la psychologie et à la pédagogie. Son ambition est de créer une plateforme visant à rassembler et à faire circuler des informations grâce à une bibliothèque, les Colloques et un bulletin de liaison. L'objectif du centre est stratégique : obtenir une voix consultative auprès des commissions de santé des organismes internationaux et obtenir des subsides en vue de recherches sur le cycle féminin. Pour le premier aspect, le père Henri de Riedmatten, habitué des colloques, observateur du Saint-Siège permanent à l'Unesco et observateur à l'OMS, est pessimiste :

Si, de fait, la direction de l'OMS dans ce domaine peut être approuvée actuellement par les catholiques, l'OMS a pris une position de neutralité, face d'une part à l'enseignement doctrinal de Pie XII, et aux pressions de grands organismes comme l'International Planning Parenthood ${ }^{25}$.

En revanche, il lui paraît plus réaliste de miser sur le fait que l'OMS et le gouvernement des États-Unis, très intéressés par les problèmes touchant à la population, orientent et subsidient très largement tous les travaux, d'où qu'ils viennent, susceptibles d'apporter des éléments de valeur sur ces problèmes. En pratique, il est donc possible de suggérer à des facultés universitaires catholiques ou à des groupes de recherche d'établir des programmes d'études dans ce domaine et ces groupes de recherche pourraient dès lors, sans avoir un statut consultatif, bénéficier d'aide puissante ${ }^{26}$. En cela, il se montre très averti des modes de financement de la recherche.

Le clergé belge sait également très bien comment actionner des bailleurs de fonds dévolus à la recherche reproductive. Depuis l'entre-deux-guerres, en effet, les universités du pays, l'Université catholique de Louvain y compris, sont largement intégrées dans les réseaux mis en place par les philanthropes américains pour accroître l'influence de leur nation dans le monde, laquelle influence s'opère par le soutien et le financement de projets de recherche, par la création et le développement d'institutions de recherche et d'enseignement et enfin par l'attribution de bourses d'études de longue durée afin de favoriser l'internationalisation des élites. Ce sont essentiellement des fonds américains qui financent la reconstruction de la bibliothèque de l'Université catholique de Louvain incendiée par les Allemands en août 191427. Pendant et après la guerre, les fondations, Ford et Rockefeller en tête, intensifient leur programme international afin de mettre en place un « plan Marshall intellectuel destiné à lutter

${ }^{24}$ Les deux organismes se séparent en 1968, vraisemblablement pour des raisons de différend linguistique (L. Tierens, 'T moet van twee kanten komen. De verhouding tussen katholicisme en wetenschap bij de oprichting van het Insitituut voor Familiale en Seksuologische Wettenschappen, Mémoire de master en histoire, Louvain, Katholiek Universiteit Leuven, 2014, p. 36).

${ }^{25} \mathrm{~V}^{\mathrm{e}}$ Colloque de Louvain, 25 et 26 mai 1963, La maîtrise sexuelle, p. 3.

${ }^{26}$ Ibid.

${ }^{27}$ K. Bertrams, « De l'action humanitaire à la recherche scientifique. Belgique, 19141930 », in L. TOURNÈs (dir.), L'Argent de l'influence. Les fondations américaines et leurs réseaux européens, Paris, Autrement, 2010, p. 45-63. 
contre le communisme en améliorant l'image des États-Unis à l'étranger ${ }^{28}$. Ce programme comprend un important volet médical avec tout un pan consacré à la gestion des populations, qui suppose la maîtrise des sciences et des techniques de la reproduction humaine. La Fondation Rockefeller et la Fondation Ford investissent dans le domaine du « contrôle de la vie » dès les années 1920 avec l'objectif de mettre en place une forme d'ingénierie sociale ${ }^{29}$. C'est la Fondation Rockefeller qui finance les travaux du zoologue Alfred Kinsey sur les comportements sexuels humains ${ }^{30}$. Dans les années 1960-1980, la Fondation Ford finance l'Université catholique de Louvain pour améliorer ses ressources personnelles, mais aussi, entre autres, pour la recherche sur la spermatogenèse $(45000 \$)$, les fonctions et la maturation post-testiculaire du sperme (300 $000 \$)$ et l'immunologie de la reproduction $(818605 \$)^{31}$.

En 1965, Suenens caresse le projet de constituer, sous les auspices de l'Université de Louvain, un Comité international qui réunirait, coordonnerait, apprécierait et diffuserait toutes les informations sur la fertilité humaine. Il veut promouvoir un programme de recherche collectif pour déterminer avec certitude la date de l'ovulation et la durée de survie des spermatozoïdes. Dans cette démarche, il se targue de l'aval du pape, qui l'encouragerait à chercher des financements par des fondations universitaires et les universités catholiques ${ }^{32}$.

À cette époque, et jusqu'à la publication de l'encyclique Humanae Vitae, les médecins catholiques se sentent autorisés à pousser très loin la recherche scientifique sur la fertilité et son corollaire, la contraception. L'ouvrage paru en 1963 de l'un des inventeurs de la pilule contraceptive, le catholique John Rock, professeur à l'Université de Harvard, The Time has come. A Catholic Doctor's Proposals to End the Battle over Birth Control, les influence beaucoup. La question qu'il pose est de savoir si on peut parler de stérilisation directe quand les progestagènes sont utilisés au service d'une régulation des naissances moralement justifiée. Selon lui, la méthode Ogino-Knauss supprime effectivement la fonction de reproduction, car, en évitant l'insémination, l'ovule ne peut être fécondé. En revanche, si l'ovulation est suspendue, il n'y a pas d'ovule à féconder. En Belgique, Jacques Ferin, chargé tous les ans aux Colloques de faire un exposé sur l'état de la recherche et expert à l'OMS, ainsi que le gynécologue campinois Ferdinand Peeters poursuivent des recherches sur la contraception et partagent les idées de $\operatorname{Rock}^{33}$.

${ }^{28}$ L. Tournès, " Carnegie, Rockefeller, Ford, Soros : Généalogie de la toile philanthropique », in L. TOURNÈs (dir.), L'Argent de l'influence. Les fondations américaines et leurs réseaux européens, Paris, Autrement, 2010, p. 5-23.

29 A. E. Clarke, Disciplining Reproduction. Modernity, American Life Sciences, and "the Problems of Sex", Berkeley, Los Angeles, Oxford, University of California Press, 1998, p. 26.

${ }^{30}$ V. L. Bullough, «The Rockefellers and Sex Research », The Journal of Sex Research, 21/2, 1985, p. 113-125 ; V. L. Bullough, « Katharine Bement Davis: Sex research and the Rockefeller Foundation », Bulletin of the History of Medicine, 62/1, 1988, p. 74-89.

${ }^{31}$ Ford Foundation records, Grants C-D, 1936-2006, Rockefeller Archival Center, [http:// dimes.rockarch.org/xtf/search].

${ }^{32} \mathrm{VII}^{\mathrm{e}}$ Colloque de Louvain, 29-30 mai 1965, La régulation des naissances, p. 74-75.

${ }^{33}$ W. Dupont, «In Good Faith. Belgian Catholics' Attempts to Overturn the Ban on Contraception (1945-1968) », in C. VANDerpelen-Diagre et C. SÄGesSer (dir.), La Sainte 
Un autre argument de Rock concerne la surpopulation qui, selon lui, menace le bien-être des hommes à l'échelle planétaire. Ce dernier argument déchire les participants des Colloques. Pour certains, la surpopulation est une « maladie du monde » qui exige de planifier les naissances au plus vite, d'autres estiment que les statistiques reposent sur des données contestables et qu'il faut surtout attendre de la science et des techniques qu'elles empêchent les pénuries alimentaires et les pandémies causées par les disettes. Ces derniers se réfèrent à l'argument religieux séculaire de la providence. Parmi ce dernier groupe, on trouve l'économiste de l'Université de Louvain Jacques Mertens de Wilmars, qui sera élu à deux reprises à la présidence de la Commission de la population de l'ONU et qui fera également partie de la Commission pontificale pour l'étude de la population, de la famille et des naissances de 1963. Il est à noter que, sur le thème spécifique de la démographie, les catholiques cherchent à se positionner. Dès 1953 est créé à Louvain un Comité provisoire international catholique des problèmes de population qui se tient en même temps que le Congrès mondial de la population et qui aboutit à la création d'un Centre de liaison scientifique des démographes catholiques établi à l'Université catholique de Louvain. Son objectif est « d'établir le contact entre les spécialistes catholiques, de promouvoir des échanges de vues et des travaux et de susciter un intérêt croissant pour les problèmes démographiques dans les milieux catholiques $\gg{ }^{34}$.

Lorsque l'on parcourt les procès-verbaux des Colloques, on est frappé par la forte influence des États-Unis, que ce soit dans le poids des stratégies géopolitiques, l'obtention de fonds ou encore les transferts de connaissance en matière médicale et scientifique, toutes disciplines confondues. Régulièrement, il est fait mention des fameux rapports du zoologue américain Alfred Kinsey, Sexual Behavior in the Human Male (1948) et Sexual Behavior in the Human Female (1953). Dans Amour et maîtrise de soi, le cardinal Suenens explique qu'il attend des scientifiques catholiques qu'ils s'approprient les résultats de l'enquête de Kinsey pour mieux les humaniser ${ }^{35}$. En 1966, un exposé du docteur français Paul Le Moal, professeur sur la sexualité féminine à l'école de formation psychopédagogique de l'Institut catholique, y fait également abondamment référence ${ }^{36}$. Cette influence s'explique évidemment par la prépondérance américaine dans le champ scientifique, mais également par une affinité de modèles. Dans le contexte de la guerre froide, le monde catholique, on le sait, se reconnaît davantage dans l'Amérique chrétienne que dans les républiques soviétiques athées. Mais il se retrouve également dans la valorisation américaine de la femme reine-du-foyer et fée-du-logis travaillant à la grandeur des valeurs de

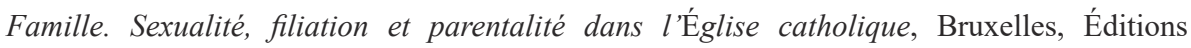
de l'Université de Bruxelles, 2017 (« Problèmes d'histoire des religions », 24), p. 67-76 et I. Brosens, The Challenge of Reproductive Medicine at Catholic Universities. Time to Leave the Catacombs, Louvain/Dudlet, Peeters, 2006.

34 J. Mertens de Wilmars, «Impression et Conclusions du Congrès mondial de La Population à Rome Septembre 1947 », Social Compass, 3, 5-6, 1956, p. 78.

35 L.-J. SuENENS, Amour et maîtrise de soi, op. cit., p. 177.

${ }^{36}$ P. Le MoAL, « Psychologie sexuelle de la femme (jusqu'à la puberté) », VIII ${ }^{e}$ Colloque de Louvain, 20-22 mai 1966, Sexualité féminine, p. 73-75. 
la liberté et de la démocratie à partir de sa cuisine ${ }^{37}$. Il est intéressant de constater que, néanmoins, l'Amérique, comme on dit à l'époque, est aussi volontiers érigée en symbole d'une érotisation outrageuse et dangereuse. Pour le docteur de Guchteneere, il y aurait une « différence de tonus érotique [...] entre les États-Unis et l'Occident ${ }^{38}$. L'affirmation d'un idéal catholique qui transcende le modèle américain s'affirme encore après 1968.

\section{La sexologie personnaliste au service de la liberté ?}

Comme on le sait, malgré l'énergie que déploient les défenseurs catholiques, à travers le monde entier en faveur d'un assouplissement de la doctrine sur la contraception, ce n'est pas cette option qui sera choisie par Paul VI. La publication le 25 juillet 1968 de l'encyclique Humanae Vitae déclenche une profonde vague de déception, voire de contestation, chez les fidèles dans la plupart des pays. Dans plusieurs d'entre eux, le clergé, afin de canaliser ce mécontentement, opte pour des déclarations proclamant la liberté de conscience des croyants ${ }^{39}$. En Belgique, le cardinal Suenens soutient la publication d'un texte épiscopal allant dans ce sens ${ }^{40}$. Par la suite, il s'éloignera du devant de la scène concernant les questions de sexualité et investira d'autres domaines religieux. Cela ne l'empêche pas de continuer à s'intéresser aux Colloques. De leur côté, les animateurs des Colloques, qui s'étaient presque exclusivement préoccupés de contraception jusqu'en 1969, investissent d'autres champs de la sexologie. Les sujets des années qui suivent vont du célibat comme état de vie à la prostitution en passant par la sexualité des handicapés et des personnes âgées. Plus généralement, le discours sur la sexualité et la sexologie, c'està-dire sur leurs finalités, tend à se modifier.

La lecture des procès-verbaux des Colloques laisse penser que, jusqu'en 1969, une communauté de vues est partagée par ses participants autour de l'absolu que représente la continence, aboutissement de la sexualité qui indique le sommet de la maturité autant du couple que de l'individu. Ainsi de ce texte du psychiatre JeanRaymond Bertolus, expert près le Tribunal de Paris pour les problèmes éducatifs et familiaux, invité régulièrement à la Commission pontificale :

Les vraies valeurs ne sont-elles pas dans un certain renoncement? Ce n'est pas la jouissance qui est facteur de promotion humaine, mais bien plus le renoncement à trouver cette jouissance. C'est par le renoncement qu'il y a plus grande autonomie, plus grande communion avec les autres et que peut s'installer un dialogue interpersonnel

${ }^{37}$ H. Lavaille, "Gender and women's rights in the Cold War », in R. H. Immerman et P. GoEdDE (dir.), The Oxford Handbook of the Cold War, Oxford, Oxford University Press, 2016, p. 523-539.

${ }^{38} \mathrm{VIII}^{\mathrm{e}}$ Colloque de Louvain, 20-22 mai 1966, op. cit., p. 104.

${ }^{39}$ Notamment : A. HARris (dir.), The Schism of '68: Catholicism, contraception and "Humanae Vitae" in Europe, 1945-1975, Cham, Suisse, Palgrave Macmillan, 2018 (« Genders and Sexualities in History ») et J. T. McGreEvy, Catholicism and American Freedom. A History, New York/Londres, W. W. Norton \& Company, 2003.

${ }^{40}$ L. DeClerCK, «La réaction du cardinal Suenens et de l'épiscopat belge à l'encyclique Humanae Vitae : Chronique d'une Déclaration (juillet-décembre 1968) », Ephemerides Theologicae Lovanienses, 84, 2008, p. 1-68. 
authentique. De même que dans l'éducation d'un enfant, qui l'amène à sa maturité d'homme, il faut des renoncements [répétés], par exemple : renoncement au complexe d'Edipe, de même, pour le couple arrivé à maturité, le renoncement à des satisfactions peut seul être le point de départ d'une sublimation et sortir un être de son isolement ${ }^{41}$.

Cependant, l'entente se fissure au fur et à mesure que les invités aux Colloques se diversifient et que, d'une certaine manière, ils se rapprochent de l'Université. À l'issue de la rencontre de l'année 1969, les organisateurs demandent aux participants de se prononcer sur le fonctionnement des Colloques. La sœur Françoise Vandermeersh, de la revue française Échanges, Française très impliquée dans le combat pour une plus grande place des femmes au sein de l'Église ${ }^{42}$, déplore que la liberté personnelle soit absente des discussions : « [N] ous ne pouvons pas nous situer librement face aux recherches de cette science [la sexologie] », explique-t-elle ${ }^{43}$. Le médecin français Bernard Vincent, parmi les initiateurs du Centre de liaison des équipes de recherche - sous-entendu sur le contrôle des naissances - de Lille ${ }^{44}$, remet, lui, explicitement en question le cadre clérical des échanges ${ }^{45}$. Quant à Robert Volcher, pourtant directeur des deux institutions chapeautant le tout à Louvain, il avoue son inconfort face à une assemblée où le regard des autorités ecclésiastiques est prédominant : " [J]e me sens jugé par plus d'un quart à ma fidélité à l'Église. ${ }^{46}$

Il est notable que ce qui est reproché aux Colloques lors de ces échanges ne soit pas l'apriori idéologique concernant la sexualité, mais la tutelle de l'Église. C'est dans l'air du temps. L' « esprit de mai 68 » comme l' « esprit de Vatican II », pour reprendre les heureuses formules de l'historien Gerd-Rainer Horn, expliquent évidemment cette remise en question de l'autorité ${ }^{47}$. On est par ailleurs à une étape charnière de l'histoire des universités catholiques. D'une part, l'internationalisation du champ scientifique impose aux universités et aux chercheurs de faire acte d'indépendance à l'égard de toute autorité politico-religieuse. D'autre part, Vatican II, et tout particulièrement la constitution pastorale Gaudium et Spes (1965), a invité les croyants à se saisir des sciences humaines comme outils d'évangélisation et à ne pas se couper des débats scientifiques qui sont les «signes du temps » auxquels le chrétien doit être attentif. Dès la fin du Concile, la Congrégation pour l'enseignement catholique s'est lancée dans un travail de réflexion pour permettre aux universités catholiques de participer à la recherche scientifique ; lequel travail aboutira à la constitution Sapientia Christiana (1979). Dans le même temps, après quelques décennies de travail théologique pour se

${ }^{41} \mathrm{VI}^{\mathrm{e}}$ Colloque de Louvain, 31-31 mai 1964, Le sens de la sexualité, p. 95.

42 S. Rousseau, Françoise Vandermeersch. L'émancipation d'une religieuse, Paris, Khartala, 2012 (« Signes des Temps »).

${ }^{43}$ Actes $d u X^{\mathrm{e}}$ Colloque international de sexologie, Louvain, 30, 31 mai et $1^{\text {er }}$ juin 1969, Orientations anciennes et récentes concernant les structures du comportement sexuel $d u$ couple, op. cit., p. 223.

${ }^{44}$ M. Sevegrand, op. cit., p. 188 et s.

${ }_{45}$ Actes $d u X I^{\mathrm{e}}$ Colloque international de sexologie, op. cit., p. 223-224.

${ }^{46}$ Ibid., p. 227.

${ }^{47}$ G.-R. Horn, The Spirit of '68: Rebellion in Western Europe and North America, 19561976, Oxford, Oxford University Press, 2007 et G.-R. Horn, The Spirit of Vatican II: Western European progressive Catholicism in the long sixties, Oxford, Oxford University Press, 2015. 
familiariser et s'adapter à la sociologie critique et à la psychanalyse, les catholiques se sont approprié ces disciplines et ont des représentants dans leurs instances de consécration $^{48}$. Dans les années 1960, pour la plupart, les académiques croyants de l'Université catholique de Louvain font le choix, soutenu par leurs autorités, d'extraire leur production scientifique d'un projet explicitement évangélique ${ }^{49}$. Cette évolution se marque très nettement dans les Colloques. La proportion d'invités théologiens et/ ou issus du clergé reste stable, mais la tutelle de l'Église s'amoindrit. De son côté, l'Institut des sciences familiales et sexologiques assoit sa légitimité scientifique, renforce ses liens avec la faculté de médecine et de psychologie et s'émancipe par rapport aux autorités religieuses. En d'autres termes, il se sécularise.

Cependant que la tutelle de l'Église s'amoindrit, d'autres normes sont renforcées. Le personnalisme ${ }^{50}$, qui guide les prises de position des théologiens et médecins de l'Université de Louvain depuis l'après-guerre ${ }^{51}$, trouve un nouveau thuriféraire en Robert Volcher, qui travaille d'une certaine manière à le séculariser. Son discours d'ouverture au Colloque de 1969 est à cet égard révélateur. Après avoir rappelé l'importance d'intégrer toutes les dimensions de l'Homme, soit le biologique, le psychologique et le sociologique, pour une appréhension adéquate des problèmes, il expose les finalités de l'acte sexuel.

La sexualité est un moyen de communication privilégié, car elle est la rencontre de deux êtres au niveau de leur totalité. La sexualité humaine dès lors implique un engagement vis-à-vis de l'autre et un échange de valeurs avec lui. Une relation harmonieuse au sein d'un couple amène un progrès et un épanouissement des personnes. La sexualité, si elle comporte la génitalité et la fécondité, ne se confond pas avec la fonction de reproduction et ne se limite pas à elle. D'où le problème de la valeur et du sens de l'acte dit sexuel par rapport à la relation du couple, par rapport à la rencontre et au dialogue des deux personnes impliquées. La sexualité se définit précisément par la rencontre, par l'aspiration à la rencontre à tous les niveaux. La génitalité ne vise que la relation physique, la sexualité vise la rencontre totale ; la génitalité mène à l'orgasme, la sexualité mène au bonheur à deux ${ }^{52}$.

Dans cette perspective, la sexologie doit être un moyen pour parvenir au « bonheur » des couples, conçus exclusivement dans une relation de type conjugale et hétérosexuelle. Au-dessus de ce projet plane un spectre, le divorce, qui, en croissance constante, inquiète le monde catholique. En dix ans, les animateurs des Colloques ont donc profondément changé de paradigme quant aux finalités de la discipline qu'ils

48 A. Desmazières, op. cit. et Sociologies catholiques, dossier spécial des Archives des sciences sociales des religions, 2017/3 ( $\mathrm{n}^{\circ}$ 179).

${ }^{49}$ K. Schelkens et J. Mettepenningen, Godfried Danneels, Biographie, Anvers, Uitgeverij Polis, 2015.

${ }^{50}$ Courant spirituel catholique lancé par le philosophe français Emmanuel Mounier dans l'entre-deux-guerres et basé sur une attention à la personne qui prime sur le principe du collectif, contrairement à ce qu'exigent les courants inspirés de saint Thomas.

${ }^{51}$ W. Dupont, "Catholicisme et évolution des mœurs sexuelles dans la Flandre belge », in A. Giami et G. Hekma (éd.), Révolutions sexuelles, Paris, La Musardine, 2015, p. 329-352.

${ }_{52} \mathrm{R}$. Volcher, "Introduction et présentation du thème », Actes du XI Colloque international de sexologie, op. cit., p. 3. 
explorent : de la sexologie essentielle à la planification licite des naissances, ils sont passés à la sexologie outil de pérennisation du couple marital. Cette modification se traduit également en langage pastoral. C'est clairement lisible dans les conclusions du Colloque de 1969 présentées par le chanoine Victor Heylen, professeur de morale à la faculté de théologie de l'Université de Louvain spécialisé dans les questions familiales et qui fut le principal rédacteur de la constitution Gaudium et Spes $^{53}$. Nourri lui aussi de personnalisme, le théologien constate que « de plus en plus la conscience moderne conteste les conceptions imposées du dehors, qui n'accrochent pas la vie personnelle sincère ». Dans ces conditions, il plaide pour un ajustement de la pastorale aux situations personnelles, ce qui suppose un dialogue constant entre les tenants des différentes conceptions de l'Homme parmi les théologiens. Et il conclut :

N'est-ce pas cette liberté de valorisation d'expression que nous avons ressentie et épousée au cours de nos échanges de vues ? Cette liberté n'est-elle pas une condition indispensable à une véritable communication ? En effet, le dialogue ne s'établit pas par les discours échangés entre une pluralité de personnes, mais par ceux échangés dans une pluralité de pensée. Les idées différentes sont nécessaires au dialogue. Puisse le choc des idées se poursuivre dans les colloques futurs ${ }^{54}$.

Dans les années qui suivent, les invités des Colloques témoignent d'une réelle volonté de discuter les tendances et discours qui concernent la sexualité ; tout en restant dans le cadre du huis clos, puisque les rencontres restent fermées au public. Peu à peu, ils délaissent tout l'appareil rhétorique de prévention morale à l'égard des œuvres profanes. Lors des derniers Colloques, les thérapies des Américains Masters et Johnson comme l'ouvrage La Révolution sexuelle de Wilhelm Reich (traduit en français en 1968) font l'objet de présentations détaillées ${ }^{55}$. Ce qui se dévoile dans la retranscription des exposés de l'époque, c'est la volonté de participer au champ scientifique de la sexologie venue, en grande partie, des États-Unis, en n'abandonnant pas les principales valeurs de l'éthique catholique de la fidélité conjugale. Les apports sont analysés pour y puiser les outils thérapeutiques et intellectuels les plus aptes à aider le travail des premiers sexologues formés par l'Institut des sciences familiales et sexologiques de Louvain.

\section{Conclusion}

L'évolution des Colloques internationaux de sexologie de Louvain témoigne de la parfaite adaptation des cadres et experts en sexualité catholiques aux normes du néolibéralisme et à l'émergence de la sexologie comme thérapie du bien-être à la fin des années 1960. Pour prendre la mesure de cette adaptation dans le sens de la sécularisation, il faut avoir en tête le caractère structurellement

${ }^{53}$ A. Houssiau, "In memoriam Mgr V. Heylen », Revue théologique de Louvain, $12^{\mathrm{e}}$ année, fasc. 2, 1981, p. 274-276.

54 V. L. Heylen, "Conclusions générales », Actes $d u X I^{\mathrm{e}}$ Colloque, op. cit., p. 213-214.

${ }^{55}$ Voir dans XVIII Colloque international de Sexologie, Louvain, 28, 29 et 30 mai 1976, Réflexions sur une sexologie pluridisciplinaire, Louvain, Centre international Cardinal Suenens, 1976 : J. Van Damme, «Sexualité et politique », p. 133-148 ; F. Vansteenwergen, " Expérience with the Masters-and-Johnson Therapy for sexual dysfunctions », p. 184-189 et E.-J. Leblanc et Cl. Gillis, « Premières considérations d'un centre de thérapie sexuelle pluridisciplinaire inspiré des méthodes mastériennes », p. 190-195. 
incompatible de la sexologie (au sens d'une discipline scientifique autonome) et de la morale catholique. Le long article consacré à la sexualité dans l'encyclopédie Catholicisme. Hier, aujourd 'hui, demain est à cet égard éclairant. Son auteur, l'abbé Gérard Mathon, doyen de la faculté de théologie de l'Université catholique de Lille et qui fut un habitué des Colloques de Louvain, y explique que la sexologie comme science autonome ne peut que très difficilement être abordée par l'Église, laquelle est incapable de concevoir la sexualité en dehors de sa finalité conjugale et procréative. Pour lui, les sexologues, qu'ils soient pédagogues ou scientifiques, sont nocifs. Dans les deux cas, ils polarisent l'attention sur la fonction érotique et organique de l'acte sexuel et le coupent de sa finalité relationnelle, laquelle est constituante de la personnalité de chaque individu. En établissant les normes de fonctionnement de la sexualité, ils la normalisent et la « confisquent». Les sciences, et plus particulièrement la biologie - Kinsey en tête -, sont accusées de ne pas réellement faire la différence entre la sexualité animale et humaine ${ }^{56}$.

Pourtant, force est de constater la remarquable pérennité des premières institutions catholiques consacrées à la sexologie en Belgique, au Québec et en Italie. Dans ces trois pays, les principaux instituts de sexologie sont à la base des organismes dédiés à l'encadrement de la vie conjugale ${ }^{57}$. Dans un premier temps, ils utilisent les données scientifiques pour rendre plus performatifs leurs prescrits à vocation religieuse. Dans un second temps, ils évacuent la morale catholique, sans forcément délaisser l'éthique chrétienne. Leur capacité à opérer la transition vers les sciences et les services de santé sécularisés tient sans doute à la vision holiste de la vie sexuelle qu'ils adoptent dès l'origine. De la sorte, ils sont particulièrement bien armés pour s'intégrer dans les infrastructures de l'État-providence qui se stabilise à l'époque. Par ailleurs, ces institutions parviennent à produire une rhétorique qui met en valeur des normes en adéquation avec celles de la société néolibérale : l'épanouissement par la sexualité, la liberté de parole et l'importance de la communication. Dans le monde catholique, ces bienfaits ne sont admis que dans le strict cadre du mariage et le divorce est un impensé. Mais la « liberté de conscience » en ce qui concerne la sexualité que choisit de valoriser une partie du clergé après (et en opposition à) Humanae Vitae s'emboîte parfaitement avec l'impératif du néolibéralisme qui impose la délibération rationnelle des individus coresponsables de la bonne marche de la politique et du principe de « gouvernementalité ${ }^{58}$. Durant la guerre froide, si l'Église catholique avait son propre

${ }^{56}$ G. Mathon, « Sexologie », in G. Mathon et G.-H. Baudry (dir.), Catholicisme. Hier, aujourd'hui, demain, vol. 13, Paris, Letouzey et Ané, 1993, p. 1204-1250.

${ }^{57}$ Cela a été très bien étudié pour le Québec : I. Perreault, « La sécularisation des discours sur la sexualité au Québec dans les années 1960 », in J.-Ph. WARrEN (dir.), Une histoire des sexualités au Québec au XX siècle, Montréal, VLB, p. 160-171 et J.-M. LARouche, Éros et Thanatos sous l'œeil des nouveaux clercs, Montréal, VLB, 1991. Pour l'Italie : Ch. Simoneldi, A. FABrizI, R. Rossi et V. Vizzari, « Storia della sessuologia in Italia », Rivista di sessuologia clinica, 2005-2, p. 7-38.

${ }^{58}$ Principe mis en lumière par M. Foucault et que M. E. SANNA a pensé en regard de la morale sexuelle de l'Église catholique contemporaine : " Le "choix rationnel" de la morale sexuelle catholique, ou les liaisons entre Église et néolibéralisme », Raison publique, 2016/1, no 20 , p. 193-207. 
agenda en matière géopolitique (imposé par le fait que le bloc de l'Est comprenait des pays comptant une majorité catholique), en matière de normes sexuelles, elle a épousé la rhétorique de la liberté du « modèle » occidental.

\section{Bibliographie}

BERTRAMs, K., « De l'action humanitaire à la recherche scientifique. Belgique, 19141930 », in Tournès, L. (dir.), L'Argent de l'influence. Les fondations américaines et leurs réseaux européens, Paris, Autrement, 2010, p. 45-63.

Boute, L., « Les Colloques de Louvain de Mgr Suenens (1959-1974) : contributions aux débats romains sur la morale conjugale? », [2016], article inédit.

Brosens, I., The Challenge of Reproductive Medicine at Catholic Universities. Time to Leave the Catacombs, Louvain/Dudlet, Peeters, 2006.

Bullough, V. L., "Katharine Bement Davis: Sex research and the Rockefeller Foundation », Bulletin of the History of Medicine, 62/1, 1988, p. 74-89.

Bullough, V. L., "The Rockefellers and Sex Research ", The Journal of Sex Research, 21/2, 1985, p. 113-125.

Casanova, J., " Globalizing Catholicism and the return to a "universal church" ", in Rudolph, S. H. et Piscatori, J. (dir.), Transitional Religion and Fading States, Boulder, Westviewpress, 1997, p. 121-143.

Clarke, A. E., Disciplining Reproduction. Modernity, American Life Sciences, and "the Problems of Sex", Berkeley, Los Angeles, Oxford, University of California Press, 1998.

Connelly, M., Fatal Misconception. The Struggle to Control World Population, Cambridge, Londres, The Belknap Press of Harvard University Press, 2008.

DECLERCK, L., «La réaction du cardinal Suenens et de l'épiscopat belge à l'encyclique Humanae Vitae : Chronique d'une Déclaration (juillet-décembre 1968) », Ephemerides Theologicae Lovanienses, 84, 2008, p. 1-68.

DeClercK, L., « Le cardinal Suenens et la question du contrôle des naissances au Concile Vatican II », Revue théologique de Louvain, 41, 2010, p. 499-518.

Desmazières, A., " La psychanalyse entre médiatisation et censure. La morale sexuelle de Marc Oraison en procès (1955-1966) », Archives de sciences sociales des religions, 163/3, 5 décembre 2013, p. 123-142.

Dupont, W., « Catholicisme et évolution des mœurs sexuelles dans la Flandre belge », in Giami, A. et Heкma, G. (éd.), Révolutions sexuelles, Paris, La Musardine, 2015, p. 329-352.

Dupont, W., « In Good Faith. Belgian Catholics' Attempts to Overturn the Ban on Contraception (1945-1968 », in VANDERPELEN-Diagre, C. et SÄGESSER, C. (dir.), La Sainte Famille. Sexualité, filiation et parentalité dans l'Église catholique, Bruxelles, Éditions de l'Université de Bruxelles, 2017, p. 67-76.

Giami, A., «Une histoire de l'éducation sexuelle en France : une médicalisation progressive de la sexualité (1945-1980) », Sexologies, 16/3, $1^{\text {er }}$ juillet 2007, p. 219-229.

HARris, A. (dir.), The Schism of '68: Catholicism, contraception and "Humanae Vitae” in Europe, 1945-1975, Cham, Suisse, Palgrave Macmillan, 2018. 
Hertzke, A. D., " The Catholic Church and Catholicism in Global politics », in HaYnes, J. (dir.), Routledge Handbook of religion and politics, Londres/New York, Routledge, 2009, p. 48-63.

Horn, G.-R., The Spirit of Vatican II: Western European progressive Catholicism in the long sixties, Oxford, Oxford University Press, 2015.

Horn, G.-R., The Spirit of '68: Rebellion in Western Europe and North America, 1956-1976, Oxford, Oxford University Press, 2007.

Houssiau, A., «In memoriam Mgr V. Heylen », Revue théologique de Louvain, 12 année, fasc. 2, 1981, p. 274-276.

LARouche, J.-M., Éros et Thanatos sous l'œeil des nouveaux clercs, Montréal, VLB, 1991.

Lavaille, H., " Gender and women's rights in the Cold War », in Immerman, R. H. et Goedde, P. (dir.), The Oxford Handbook of the Cold War, Oxford, Oxford University Press, 2016, p. 523-539.

Mathon, G., "Sexologie », in Mathon, G. et Baudry, G.-H. (dir), Catholicisme. Hier, aujourd'hui, demain, vol. 13, Paris, Letouzey et Ané, 1993, p. 1204-1250.

MCClory, R., Rome et la contraception : Histoire secrète de l'encyclique Humanae Vitae, Paris, Éditions de l'atelier, Éditions ouvrières, 1998.

McGreevy, J. T., Catholicism and American Freedom. A History, New York/Londres, W. W. Norton \& Company, 2003.

Mertens de Wilmars, J., "Impression et Conclusions du Congrès mondial de La Population à Rome Septembre 1947 », Social Compass, 3, 5-6, 1956, p. 78.

Perreault, I., " La sécularisation des discours sur la sexualité au Québec dans les années 1960 », in WARREN, J.-Ph. (dir.), Une histoire des sexualités au Québec au $X X^{\mathrm{e}}$ siècle, Montréal, VLB, p. 160-171.

Christens, R., " De orthodoxie van het zaad », in Wils, K. (dir.), Het lichaam, Louvain, Universitaire Pers Leuven, 2001, p. 241-245.

Rousseau, S., Françoise Vandermeersch. L'émancipation d'une religieuse, Paris, Khartala, 2012.

SAnNa, M. E. «Le "choix rationnel" de la morale sexuelle catholique, ou les liaisons entre Église et néolibéralisme », Raison publique, 2016/1, n 20, p. 193-207.

Schelkens, K. et Mettepenningen, J., Godfried Danneels, Biographie, Anvers, Uitgeverij Polis, 2015.

Sevegrand, M., Les Enfants du bon Dieu. Les catholiques français et la procréation au XX $X^{\mathrm{e}}$ siècle, Paris, Albin Michel, coll. « Histoire », 1995.

Simonelli, Ch., Fabrizi, A., Rossi, R. et Vizzari, V., « Storia della sessuologia in Italia », Rivista di sessuologia clinica, 2005, 2, p. 7-38.

SHANI, G., " Transnational religious actors and international relations », in HaYNES, J. (dir.), Routledge Handbook of religion and politics, Londres/New York, Routledge, 2009, p. 308-322.

Sociologies catholiques, dossier spécial des Archives des sciences sociales des religions, 2017, 3, $\mathrm{n}^{\circ} 179$.

SoHn, A.-M., « Les catholiques français entre abstinence et "apaisement de la concupiscence" ", Sexual Cultures in Europes. Themes in sexuality, edited by Franz Eder, Leslie Hall, Gert Hekma, 1996, p. 233-254. 
Suenens, L.-J., « Christianisme et santé », Collectanea Mechliniensia, novembre 1958, p. 10.

SuEnens, L.-J., Amour et maîtrise de soi. Un problème crucial, [Paris-Bruges], Desclée de Brouwer, 1960.

TIERENS, L., 'T moet van twee kanten komen. De verhouding tussen katholicisme en wetenschap bij de oprichting van het Insitituut voor Familiale en Seksuologische Wettenschappen, Mémoire de master en histoire, Louvain, Katholiek Universiteit Leuven, 2014.

Tournès, L., "Carnegie, Rockefeller, Ford, Soros : Généalogie de la toile philanthropique », in Tournès, L. (dir.), L'Argent de l'influence. Les fondations américaines et leurs réseaux européens, Paris, Autrement, 2010, p. 5-23.

\section{Fonds d'archives}

Fonds de l'Institut des sciences familiales et sexologiques, Louvain-la-Neuve, Archives de l'Université catholique de Louvain.

Colloque de Louvain, 1959-, Archives de l'Université catholique de Louvain, FI 69.

Ford Foundation records, Grants C-D, 1936-2006, Rockefeller Archival Center. [http://dimes.rockarch.org/xtf/search]. 